\title{
An exploratory study of the patients' sleep patterns and inflammatory response following cardiopulmonary bypass (CPB)
}

\author{
Jesus M Casida, Jean E Davis, LaVonne Shpakoff and Hossein Yarandi
}

\begin{abstract}
Aims and Objectives. To describe sleep patterns and inflammatory response postCPB, determine sleep pattern changes and inflammatory response over time and explore relationships between sleep and biomarkers of stress and inflammation.

Background. Despite the numerous citations of the role of sleep in restoration and health maintenance, a paucity of research exists about this phenomenon in patients undergoing CPB. Specifically, there is no research that has explored correlations between sleep patterns and systemic inflammatory response in adult cardiac surgery patients.

Design. Exploratory, repeated-measures, correlational study.

Method. Subjects were recruited from a Midwestern urban hospital. Of the 25 eligible subjects, 16 males and four females completed the study. Wrist actigraphy was used to measure sleep variables. Salivary cortisol and C-reactive protein (C-RP) levels were measured daily. Data were collected during postoperative nights/days 1 through 4 (T1-T4).

Results. Subjects' sleep onset latency (SOL) median scores (0 minute) were within normal range across time periods, whereas median scores for wake after sleep onset (WASO > 270 minutes), sleep fragmentation index (SFI >51\%), total sleep time ( $<153$ minutes) and sleep efficiency index (SEI $<36 \%$ ) fell outside the normal ranges. Changes in the median sleep scores over time, however, were not significant at $p>0 \cdot 05$. Median cortisol levels were within normal range $(0 \cdot 3-0 \cdot 8 \mu \mathrm{g} / \mathrm{dl})$ from T1-T4, but the C-RP level peaked at T2 (median $=2370 \mathrm{pg} / \mathrm{ml})$. Strong correlations were found: (1) between SFI-cortisol $\left(r_{s}=0.82\right)$, C-RP $\left(r_{s}=0.65\right)-$ WBC $\left(r_{s}=0.69\right)$; (2) between SEI-C-RP $\left(r_{s}=0.58\right)$; (3) between WASO-WBC $\left(r_{s}=0.48\right)$, WASO and cross-clamp time $\left(r_{s}=0.50\right)$; and (4) between SOL-age $\left(r_{s}=-0.55\right)$ at $p<0.05$.

Conclusions. Subjects were severely sleep-deprived with inflammatory response exaggerations warranting further investigations using larger sample sizes.

Relevance to clinical practice. This study offers a foundation for developing a conceptual model explaining mechanisms of sleep disturbance and inflammatory response postCPB. This knowledge is crucial for testing sleep-promoting interventions to modulate inflammatory responses essential for preventing complications, and restoring health.
\end{abstract}

What does this paper contribute to the wider global clinical community?

- Worldwide, sleep pattern disturbance is prevalent in adults who have undergone cardiac surgery. Further research is warranted to closely examine the relationship between patients' systemic inflammatory response and sleep disturbance. Additionally, future research should also aim to determine the effects of inflammatory response on patients' sleep pattern, postoperative recovery, complication(s) associated with cardiopulmonary bypass and health outcomes.

- Cardiac surgical nurses throughout the world are well positioned to lead the advancement of sleep promotion science in cardiac surgery. They have the power to transform the night-time care delivery processes in cardiac surgical intensive and progressive care units grounded by theoretical and empirical knowledge.
Authors: Jesus M Casida, PhD, CCRN-CSC, Assistant Professor, Division of Acute, Critical and Long-Term Care, The University of Michigan School of Nursing, Ann Arbor, MI; Jean E. Davis, PhD, $\mathrm{RN}$, Associate Dean for Academic and Clinical Affairs, Wayne State University College of Nursing, Detroit, MI; LaVonne Shpakoff, MSN, PhD in Nursing Education (candidate), Eastern Michigan University School of Nursing, Ypsilanti, MI; Hossein
Yarandi, PhD, Professor, Wayne State University College of Nursing, Detroit, MI, USA

Correspondence: Jesus M Casida, Assistant Professor, Division of Acute, Critical and Long-Term Care, The University of Michigan School of Nursing, 400 North Ingalls, Room 2174, Ann Arbor, MI 48109, USA. Telephone: +1 3132047665.

E-mail: jcasida@umich.edu 
Key words: Adult cardiac surgery, biomarkers, cardiopulmonary bypass, inflammation, sleep patterns, stress

Accepted for publication: 18 October 2013

\section{Introduction}

Despite the numerous citations of the positive impact of restorative sleep on health risk reduction and maintenance (Vester et al. 2008), there exists a paucity of research about the sleep patterns among cardiac surgery patients who have undergone a $\mathrm{CPB}$, commonly referred to as a heart-lung machine (Casida et al. 2011). The majority of the published research on sleep has been centred on community-dwelling adults with various types of health conditions and diseases. Disturbances in the sleep patterns among adults with asthma, cancer, cardiac and vascular conditions, chronic obstructive pulmonary disease, fibromyalgia, or psychiatric and behavioural disorders have been linked to stress and inflammation (Vester et al. 2008, Mullington et al. 2009, 2010, Parish 2009). This phenomenon, however, is understudied in acutely and critically ill patients, specifically in cardiac surgery.

Although research on sleep in the acute and critical care settings is limited, there is a consistent finding that sleep pattern disturbances are prevalent in adult cardiac surgery patients due to behavioural, psychological and environmental factors (Casida \& Lemanski 2010, Liao et al. 2011). However, the context of sleep patterns and systemic stress and inflammation (i.e. inflammatory response) from $\mathrm{CPB}$ during the immediate postoperative or recovery period (Bojar 2011) warrants an empirical evaluation. Equally important is the need to develop an empirically based conceptual framework mapping the linkages between sleep patterns, systemic inflammatory response and other aetiological factors for sleep disruptions to delineate which problematic areas are amenable for intervention. Nurses are well positioned to develop and test interventions aimed to modulate or reduce the systemic inflammatory response postCPB to reduce risks or prevent postoperative complications.

\section{Background}

A recent systematic review of the literature showed that the number of published research articles about the sleep patterns of adult cardiac surgery patients is small when compared to the vast amount of research on sleep that has been conducted in other patient populations (Liao et al. 2011).
Although the majority of the instruments used in these studies were self-report questionnaires, a recurring theme suggests that sleep disturbances are a common problem and a cause of care dissatisfaction among patients recovering from cardiac surgery. Postoperative pain, anxiety, noise, bright lights, frequent measurements of vital signs and hemodynamic parameters, laboratory tests, and physical care (e.g. bed bath) provided during the night or very early morning hours were commonly reported causes of sleep disturbances in cardiac surgical care units (Casida \& Nowak 2011). Sleep patterns of patients who have undergone cardiac surgery are characterised by prolonged sleep onset, fragmented sleep, multiple awakenings, shortened sleep time and poor sleep efficiency (Liao et al. 2011). Consequently, patients demonstrate daytime sleepiness, fatigue, irritability, susceptibility to confusion or delirium, resulting in lack of motivation to engage in performing postoperative activities such as use of flow-oriented spirometry and ambulation. Sleep disruptions have also been associated with delayed recovery periods and longer hospitalisation stays (Casida \& Lemanski 2010, Casida \& Nowak 2011).

In cardiac surgery, the exposure of the body to $\mathrm{CPB}$ creates a cascade of stress response and inflammatory processes, predisposing a patient to a myriad of postoperative complications such as arrhythmias, metabolic disturbances, stroke and delayed wound healing, among others (Bojar 2011). Theoretically, an altered sleep-wake cycle combined with the effects of $\mathrm{CPB}$ can exaggerate the release of stress hormones (e.g. cortisol) and inflammatory substances (e.g. C-reactive protein), which may increase the incidence of postoperative complications. Thus, the purpose of this study was to initiate the process of validating the assumption that the stress and inflammation following $\mathrm{CPB}$ may be exaggerated by sleep disturbances. Specific aims of this study were: (1) to describe the patterns of sleep onset latency (SOL), wake after sleep onset (WASO), sleep fragmentation index (SFI), total sleep time (TST) and sleep efficiency index (SEI), and the biomarkers for stress (cortisol) and inflammation (C-reactive protein) postCPB, (2) to determine the changes in the patterns of sleep and biomarker variables from postoperative nights/days $1-4$, and (3) to explore the relationship between sleep pattern characteristics and biomarker variables. 


\section{Methods}

\section{Study design and sample}

Based on the specific aims of the study, we employed an exploratory, repeated-measures correlational design. Prior to the implementation of this study, appropriate institutional review board approvals were obtained. Subjects' eligibility to participate in this study was based on the following inclusion criteria: (1) adult male or female aged 18 years or older, (2) scheduled for first-time elective cardiac surgery using $\mathrm{CPB},(3)$ able to read, write and understand English, and (4) provided an informed consent. Subjects were excluded if they had a documented history or diagnosis of sleep disorders regardless of duration and aetiology, migraine headaches and/or behavioural (psychiatric) disorders. Subjects were randomly selected from the list of patients scheduled for an elective cardiac surgery. Randomisation was accomplished by selecting subjects with a medical record number ending with an odd number. Of 91 subjects who were screened, 25 agreed to participate and subsequently provided their informed consents. This study was implemented at a large, teaching hospital located in Southeast Michigan, USA.

\section{Variables and measures}

\section{Primary variables}

Sleep patterns. For the purposes of this exploratory study, an objective measure of sleep patterns (e.g. wrist actigraphy) was employed. A wrist actigraphy has an accelerometer that detects and analyses patient movements which are then converted into sleep pattern data by computer software. The Actiwatch 64W (Respironics, Bend, Oregon, USA) was used to measure the subjects' (1) $S O L$ (time between going to bed and the onset of sleep), (2) WASO (time spent awake from sleep onset to final awakening), (3) SFI (disruption of continuous sleep by awakenings), (4) TST (total sleep period minus the time spent awake during the sleep period) and (5) SEI (ratio of TST and time spent in bed). Wrist-actigraphically measured sleep pattern variables (e.g. SFI) have shown adequate reliability, specificity and sensitivity when compared to the sleep variables measured using polysomnography (gold standard of sleep measurement) among adults with normal or abnormal sleep patterns (Ancoli-Israel et al. 2003). Normal values for TST and WASO scores vary across the lifespan, but the normal range for wrist-actigraphically measured $\mathrm{SOL}$ is $<15$ minutes, SEI is $>85 \%$ and SFI is $\leq 49 \%$ in the adult population (Aubert-Tulkens et al. 1987, Ancoli-Israel et al. 2003).
Stress and inflammation. Salivary cortisol and C-reactive proteins (C-RP) were selected as biological measures for stress and inflammation, respectively. These biomarkers are common indicators in measuring responses to surgical stress and acute injury, specifically in cardiac surgery (Cappabianca et al. 2006, Hoda et al. 2006, Christ-Crain et al. 2007). High correlations between salivary and serum cortisol levels have been found in both human and animal subjects (Rao et al. 2010, Estrada-Y-Martin \& Orlander 2011). Salivary cortisol was measured using high-sensitivity enzyme-linked immunoassay [ELISA] (No. 1-3102, Salimetrics, LLC, State College, PA, USA). All unknowns were run in duplicate, and high and low salivary cortisol controls were run with each assay. The final optical density was read on a standard plate reader at $450 \mathrm{~nm}$. The normal 'morning' range for the salivary cortisol was $0.094-1.551 \mu \mathrm{g} / \mathrm{dl}$.

Salivary C-RP was measured with ELISA (No. 13302, Salimetrics, LLC). Also, all unknowns were run in duplicate, and high and low salivary C-RP controls were run with each assay. The final optical density was read on a standard plate reader at $450 \mathrm{~nm}$. The normal range for the salivary C-RP level was $1800 \cdot 76-2181.35 \mathrm{pg} / \mathrm{ml}$. All of these ranges were based on the intra- and interassay precision calculations reported by Salimetrics (2010), LLC.

Surrogate markers of systemic inflammatory response routinely measured postCPB were collected. These include daily white blood cell count (WBC), mean 24-hour arterial blood pressure (MAP), heart rate (HR) and serum blood glucose (BG) level (Bojar 2011). These markers were measured with the standard physiological monitoring and point-of-care testing equipment used in acute and critical care settings in the USA. In the adult population, the normal ranges for these markers are as follows: WBC $4.5-10.0 \mathrm{~mm}^{3}$, MAP 70 $110 \mathrm{mmHg}$, HR $80-100$ beats per min and glucose $70-100 \mathrm{mg} / \mathrm{dl}$ (Bojar 2011). Other surrogate markers such as cardiac output and body temperature were not collected due to variability in the instruments used and removal of pulmonary artery catheters during the study period.

\section{Secondary variables}

Pain and anxiety. The subjects' pain intensity and anxiety level were assessed using a 0 -to-10 numeric rating scale $(0=$ no pain or anxiety, $10=$ worst pain or anxiety). These assessment scales are widely used in clinical practice and research involving adult cardiac surgery patients (Casida $\&$ Lemanski 2010).

Demographic and clinical characteristics. Subjects' demographics such as age, gender, race, education, health history, among others were recorded. Additionally, com- 
mon clinical variables reported in cardiac surgery research were also recorded. These include surgical procedure, anaesthesia time (from induction to reversal of anaesthetic agents), cross-clamp and bypass times, complications, and medications routinely administered postcardiac surgery [cross-clamp time refers to the period of intentional cardiac arrest to ensure a bloodless field during surgery, while bypass time is the total duration of the patient's exposure to CPB machine (Bojar 2011)].

\section{Data collection}

Data were collected during postoperative days/nights one (T1), two (T2), three (T3) and four (T4). The subjects' SOL, WASO, SFI, TST and SEI were measured from 22:00-08:00 hours during postoperative nights $1-4$. Using salivary swabs, salivary cortisol and C-RP levels were collected daily between 06:00-08:00 hours on postoperative days $1-4$. Within 30 minutes of collection, saliva specimens were stored in a freezer at a temperature of $-56.7^{\circ} \mathrm{C}$ $\left(-70 \cdot 0^{\circ} \mathrm{F}\right)$. The subjects' pain and anxiety were measured at 22:00 hours during postoperative nights $1-4$. Demographics and clinical variables as well as surrogate markers of systemic inflammatory response were collected from the subjects' medical records at T1 through T4.

\section{Data analyses}

Of the 25 subjects who provided informed consent, 20 $(80 \%)$ provided complete data. Subjects' attrition was primarily related to postoperative stroke $(n=1)$, reoperation and renal failure $(n=1)$, cognitive dysfunction $(n=2)$ and death $(n=1)$. The sleep pattern data of the 20 subjects were analysed and scored based on procedures recommended by Respironics (2008), Bend, Oregon, USA. Saliva specimens (cortisol and C-RP levels) were thawed at room temperature and then analysed with ELISA as previously described (Salimetrics 2009, 2010). Data were managed and analysed using IBM sPsS, version 19.0 (Armonk, NY, USA) and SAS, version 9.0 statistical software (Cary, NC, USA).

Descriptive statistics were used to calculate frequency distributions, means, standard deviations, quartiles, and medians of primary and secondary variables. Normality and abnormality of data distributions were evaluated using descriptive statistical procedures including visual examination of a histogram. All continuous variables were normally distributed with the exception of sleep patterns, cortisol and C-RP variables (Pearson's skewness coefficient $>-1 \cdot 0$ to 1.0; Munro 2001). Parametric and nonparametric statis- tics and mixed model were employed. A general estimating equation (GEE) model (ProcGenmod with SAS) was used to detect the changes in the subject's sleep patterns, cortisol and C-RP variables over time (T1-T4). Likewise, multivariate repeated-measures analysis of variance (MR-MANOvA) was used to detect the changes in the subject's WBC, MAP, HR and BGs over time. Analysis of covariance was used to determine whether pain and anxiety levels, medications, and other clinical data had any influence on the sleep pattern, biomarkers and surrogate markers of stress/inflammation results. Finally, Spearman's rank correlations were used to determine the relationship between the sleep patterns, biomarkers and surrogate markers of stress/inflammation variables. Statistical significance was set a priori at a level of $p<0.05$ (two-tailed tests).

\section{Results}

\section{Sample characteristics}

The demographics and clinical profiles of the 20 subjects who completed the study are summarised in Table 1 . The majority of the subjects were White males; most were married, had a high school education and were unemployed due to retirement or disability. Common preoperative risk factors among the subjects were tobacco smoking, coronary artery disease and hypercholesterolaemia. Intraoperatively, all patients underwent a $\mathrm{CPB}$ with a total intraoperative average time of 328 minutes, which is the time of induction up to reversal of anaesthetic agents. None of the subjects had intraoperative complications, but $30 \%$ developed postoperative arrhythmias (sinus tachycardia, premature atrial contractions or atrial fibrillation) treated with standard pharmacological agents. While all subjects received narcotic or non-narcotic analgesic agents for pain control, only $15 \%$ required anxiolytic medications throughout the study period (Table 1).

\section{Sleep and systemic inflammatory response postCPB}

Subjects' SOL was within normal range of $<15$ minutes (median $=0$ minute $)$ throughout the study time periods. At $\mathrm{T} 4$, the upper quartile (Q3) of the study subjects' SOL score was 24 minutes. They spent the majority of the sleep time awake on most nights as shown by WASO median scores of 270-318 minutes and TST median scores of 132153 minutes. Moreover, the subjects' median SFI scores were high $(>50 \%)$ and the median SEI scores were low $(<36.0 \%)$. GEE showed no significant differences within subjects' median sleep scores and time effects (T1-T4) on 
Table 1 Sample Characteristics

\begin{tabular}{|c|c|}
\hline Demographics and clinical data & $n(\%) *$ \\
\hline Age (mean in years) & $58 \cdot 5 \pm 17 \cdot 0$ \\
\hline \multicolumn{2}{|l|}{ Gender } \\
\hline Male & $16(80)$ \\
\hline Female & $4(20)$ \\
\hline \multicolumn{2}{|l|}{ Marital Status } \\
\hline Single & $4(20)$ \\
\hline Married & $14(70)$ \\
\hline Divorced & $1(5)$ \\
\hline Widow & $1(5)$ \\
\hline \multicolumn{2}{|l|}{ Race } \\
\hline Blacks & $5(25)$ \\
\hline Whites & $15(75)$ \\
\hline \multicolumn{2}{|l|}{ Education } \\
\hline High School & $7(35)$ \\
\hline Some College & $4(20)$ \\
\hline Baccalaureate degree or higher & $2(10)$ \\
\hline \multicolumn{2}{|l|}{ Employment Status } \\
\hline Employed & $7(35)$ \\
\hline Unemployed (Retired/Disabled) & $10(50)$ \\
\hline \multicolumn{2}{|l|}{ Social History } \\
\hline Tobacco smoking & $8(40)$ \\
\hline Alcohol consumption & $7(35)$ \\
\hline \multicolumn{2}{|l|}{ Medical History } \\
\hline Coronary artery disease & $11(55)$ \\
\hline Heart failure/valvular disease & $6(30)$ \\
\hline Diabetes & $8(40)$ \\
\hline Hypercholesterolaemia & $10(50)$ \\
\hline Pulmonary disease & $3(15)$ \\
\hline Other (e.g. vascular diseases) & $7(35)$ \\
\hline \multicolumn{2}{|l|}{ Surgical History (noncardiac) } \\
\hline Yes & $14(70)$ \\
\hline No & $6(30)$ \\
\hline \multicolumn{2}{|l|}{ Surgical Procedure } \\
\hline Coronary artery bypass graft (CABG) & $12(60)$ \\
\hline Valvular repair or replacement & $2(10)$ \\
\hline CABG + valvular repair or replacement & $6(30)$ \\
\hline Anaesthesia time (mean in min) & $327 \cdot 9 \pm 98.4$ \\
\hline Cross-clamp time (mean in min) & $96 \cdot 9 \pm 53 \cdot 7$ \\
\hline Bypass time (mean in min) & $134 \cdot 9 \pm 54 \cdot 0$ \\
\hline \multicolumn{2}{|l|}{ Postoperative complications } \\
\hline Cardiac arrhythmias & $6(30)$ \\
\hline Pulmonary atelectasis & $2(10)$ \\
\hline Infection & $1(5)$ \\
\hline \multicolumn{2}{|l|}{ Medications } \\
\hline Anxiolytics & $3(15)$ \\
\hline Beta-blockers & $20(100)$ \\
\hline Inotropic agents & $2(10)$ \\
\hline Narcotic analgesics & $20(100)$ \\
\hline Non-narcotic analgesics & $10(50)$ \\
\hline Sleep medications & $1(5)$ \\
\hline Vasoactive agents & $10(50)$ \\
\hline Anxiety level (mean) & $2 \cdot 4 \pm 2 \cdot 1$ \\
\hline Pain level (mean) & $3 \cdot 3 \pm 1 \cdot 7$ \\
\hline
\end{tabular}

*Because of rounding or missing data, not all percentages total 100 ( \pm standard deviation). their sleep patterns. Also, the changes in the median sleep scores over time were not significant at $p<0.05$ (Table 2).

The subjects' salivary C-RP level was high at T2 $($ median $=2370 \mathrm{pg} / \mathrm{ml})$, while the median salivary cortisol levels remained within normal range $(0.3-0.8 \mu \mathrm{g} / \mathrm{dl})$ at T1-T4. A consistent decline in the cortisol levels across time periods, from a median of $0.8 \mu \mathrm{g} / \mathrm{dl}$ at $\mathrm{T} 1$ to $0.3 \mu \mathrm{g} / \mathrm{dl}$ at T4, was noted in contrast with C-RP levels which fluctuated across time periods (median C-RP level ranges from $1503 \mathrm{pg} / \mathrm{ml}$ (T3)-2370 (T2) as shown in Table 2). Although there was a decline in the cortisol levels from T1 to T4 along with a decline in C-RP levels from T2-T4, a consistent increase in the surrogate markers of systemic inflammatory response (WBC and $\mathrm{BG}$ ) along with normal ranges of physiological markers (ABP and $\mathrm{HR}$ ) was noted throughout the study time periods (Tables 2 and 3). Results of GEE showed no significant differences within subjects' median scores and the effects of time on cortisol and C-RP levels over time (Table 2). MR-MANOva also showed no significant differences within subjects' mean scores and the effects of time on surrogate markers of stress and inflammation over time (Table 3 ).

\section{Relationships between sleep patterns and inflammatory response}

Table 4 offers a summary of the correlations between sleep patterns, biomarkers and surrogate markers of stress/inflammation variables postCPB. Strong, positive correlations were found between the median scores of SFI and cortisol $\left(r_{s}=0.82, \quad p<0.001\right), \quad$ between SFI-C-RP $\quad\left(r_{s}=0.65\right.$, $p=0.022)$, and between SFI-WBC $\left(r_{s}=0.69, p=0.029\right)$. Similar correlations were also found between the median scores of SEI and C-RP $\left(r_{s}=0.58, p=0.015\right)$ and between WASO-WBC $\left(r_{s}=0.48, p=0.031\right)$. Analyses of covariances showed that the subjects' demographic and clinical variables did not significantly explain the correlations or the outcome of sleep patterns and inflammatory response variables - biologically and physiologically. However, a strong positive correlation between cross-clamp time and WASO $\left(r_{s}=0.50, p=0.039\right)$ and a strong negative correlation between SOL and age $\left(r_{s}=-0.55, p=0.012\right)$ were found.

\section{Discussion}

Our data support the extant knowledge that systemic inflammation and hyperglycaemia are common clinical problems during the immediate recovery period postCPB (Bojar 2011). Also, the data support the role of sleep disturbances in the inflammatory response postCBP, although the extent 
Table 2 Descriptive statistics and GEE analysis of the time effects on sleep patterns, cortisol and C-RP postCPB

\begin{tabular}{|c|c|c|c|c|c|c|}
\hline Variables & $\mathrm{T} 1$ & $\mathrm{~T} 2$ & T3 & T4 & $\chi^{2}$ & $p$ \\
\hline \multicolumn{7}{|c|}{ Sleep onset latency $(\min )$} \\
\hline Q1 & $0 \cdot 0$ & $0 \cdot 0$ & $0 \cdot 0$ & $0 \cdot 0$ & \multirow[t]{4}{*}{$3 \cdot 89$} & \multirow[t]{4}{*}{$0 \cdot 27$} \\
\hline Q2 & $0 \cdot 0$ & $0 \cdot 0$ & $0 \cdot 0$ & $0 \cdot 0$ & & \\
\hline Q3 & $0 \cdot 0$ & $0 \cdot 0$ & $0 \cdot 0$ & $24 \cdot 0$ & & \\
\hline Min-Max & $0 \cdot 0-25$ & $0 \cdot 0-38$ & $0 \cdot 0-11$ & $0 \cdot 00-14$ & & \\
\hline \multicolumn{7}{|c|}{ Wake after sleep onset (min) } \\
\hline Q1 & $242 \cdot 2$ & $265 \cdot 8$ & $186 \cdot 1$ & $213 \cdot 7$ & \multirow[t]{4}{*}{$5 \cdot 12$} & \multirow[t]{4}{*}{$0 \cdot 16$} \\
\hline Q2 & $303 \cdot 5$ & $317 \cdot 9$ & $270 \cdot 1$ & $309 \cdot 4$ & & \\
\hline Q3 & $366 \cdot 0$ & $341 \cdot 7$ & 343.4 & $360 \cdot 1$ & & \\
\hline Min-Max & $68 \cdot 9-404 \cdot 8$ & $134 \cdot 6-395 \cdot 4$ & $95 \cdot 8-391 \cdot 6$ & $86 \cdot 2-404 \cdot 1$ & & \\
\hline \multicolumn{7}{|c|}{ Sleep fragmentation index $(\%)$} \\
\hline Q1 & $34 \cdot 2$ & $39 \cdot 8$ & $44 \cdot 6$ & $30 \cdot 0$ & \multirow[t]{4}{*}{$1 \cdot 64$} & \multirow[t]{4}{*}{0.65} \\
\hline Q2 & $59 \cdot 6$ & $50 \cdot 7$ & $54 \cdot 3$ & $53 \cdot 4$ & & \\
\hline Q3 & $84 \cdot 8$ & $64 \cdot 8$ & $78 \cdot 5$ & $77 \cdot 0$ & & \\
\hline Min-Max & $20 \cdot 6-130 \cdot 9$ & $17 \cdot 7-120 \cdot 1$ & $19 \cdot 8-137 \cdot 6$ & $13 \cdot 7-110 \cdot 4$ & & \\
\hline \multicolumn{7}{|c|}{ Total sleep time (min) } \\
\hline Q1 & $112 \cdot 1$ & $107 \cdot 9$ & $115 \cdot 5$ & $100 \cdot 0$ & \multirow[t]{4}{*}{$2 \cdot 80$} & \multirow[t]{4}{*}{0.42} \\
\hline Q2 & $132 \cdot 1$ & $147 \cdot 4$ & $152 \cdot 6$ & $152 \cdot 8$ & & \\
\hline Q3 & $263 \cdot 3$ & $198 \cdot 4$ & $217 \cdot 5$ & $183 \cdot 2$ & & \\
\hline Min-Max & $39 \cdot 0-350 \cdot 1$ & $20 \cdot 2-340 \cdot 9$ & $65 \cdot 5-357 \cdot 2$ & $76 \cdot 0-280 \cdot 5$ & & \\
\hline \multicolumn{7}{|c|}{ Sleep efficiency index $(\%)$} \\
\hline Q1 & $24 \cdot 5$ & $24 \cdot 6$ & $25 \cdot 8$ & $23 \cdot 8$ & \multirow[t]{4}{*}{$2 \cdot 15$} & \multirow[t]{4}{*}{$0 \cdot 54$} \\
\hline Q2 & $28 \cdot 9$ & $32 \cdot 4$ & $35 \cdot 4$ & $33 \cdot 9$ & & \\
\hline Q3 & $48 \cdot 9$ & $41 \cdot 8$ & $45 \cdot 2$ & $42 \cdot 9$ & & \\
\hline Min-Max & $11 \cdot 5-72 \cdot 8$ & $18 \cdot 6-71 \cdot 5$ & $19 \cdot 2-66 \cdot 3$ & $15 \cdot 6-75 \cdot 5$ & & \\
\hline \multicolumn{7}{|c|}{ Cortisol $(\mu \mathrm{g} / \mathrm{dl})$} \\
\hline Q1 & $0 \cdot 5$ & $0 \cdot 2$ & $0 \cdot 2$ & $0 \cdot 2$ & \multirow[t]{4}{*}{$4 \cdot 09$} & \multirow[t]{4}{*}{$0 \cdot 25$} \\
\hline Q2 & $0 \cdot 8$ & $0 \cdot 3$ & $0 \cdot 4$ & $0 \cdot 3$ & & \\
\hline Q3 & $2 \cdot 0$ & $0 \cdot 6$ & $0 \cdot 6$ & $2 \cdot 0$ & & \\
\hline Min-Max & $0 \cdot 02-6 \cdot 3$ & $0 \cdot 04-17 \cdot 3$ & $0 \cdot 05-3 \cdot 9$ & $0 \cdot 02-2 \cdot 2$ & & \\
\hline \multicolumn{7}{|c|}{ C-reactive protein $(\mathrm{pg} / \mathrm{ml})$} \\
\hline Q1 & $329 \cdot 2$ & 1771.4 & $1135 \cdot 0$ & $577 \cdot 0$ & \multirow[t]{4}{*}{$3 \cdot 33$} & \multirow[t]{4}{*}{$0 \cdot 34$} \\
\hline Q2 & $2093 \cdot 6$ & $2370 \cdot 0$ & $1503 \cdot 5$ & $1840 \cdot 1$ & & \\
\hline Q3 & 4589.7 & $3196 \cdot 3$ & $3793 \cdot 3$ & $4695 \cdot 3$ & & \\
\hline Min-Max & $40 \cdot 0-13196 \cdot 3$ & $40 \cdot 3-14357 \cdot 0$ & $77 \cdot 1-14366 \cdot 0$ & $32 \cdot 3-5439 \cdot 0$ & & \\
\hline
\end{tabular}

T1-T4 denote immediate postoperative days/nights, Q1 (lower quartile), Q2 (median), Q3 (upper quartile), Min-Max (minimum and maximum median scores).

Table 3 Descriptive statistics and MR-MANOVA of the time effects on surrogate markers of stress and inflammation postCPB

\begin{tabular}{|c|c|c|c|c|c|c|c|}
\hline \multirow[b]{2}{*}{ Variables } & \multicolumn{4}{|c|}{ Means, Standard Deviations $( \pm)$, Minimum and Maximum } & \multirow[b]{2}{*}{$F$} & \multirow[b]{2}{*}{$p$} & \multirow[b]{2}{*}{$p^{2}$} \\
\hline & $\mathrm{T} 1$ & $\mathrm{~T} 2$ & $\mathrm{~T} 3$ & $\mathrm{~T} 4$ & & & \\
\hline \multirow[t]{2}{*}{ Mean arterial blood pressure $(\mathrm{mmHg})$} & $81 \cdot 0(10 \cdot 3)$ & $84.4(10 \cdot 7)$ & $83 \cdot 5(8 \cdot 3)$ & $84.5(11.8)$ & \multirow[t]{2}{*}{0.58} & \multirow[t]{2}{*}{$0 \cdot 65$} & \multirow[t]{2}{*}{$0 \cdot 20$} \\
\hline & 68-112 & $71-111$ & $72-104$ & 73-106 & & & \\
\hline \multirow[t]{2}{*}{ Blood glucose (mg/dl) } & $144 \cdot 6(44 \cdot 2)$ & $143 \cdot 2(28 \cdot 5)$ & $136 \cdot 9(39 \cdot 5)$ & $125 \cdot 0(22 \cdot 0)$ & \multirow[t]{2}{*}{$1 \cdot 22$} & \multirow[t]{2}{*}{$0 \cdot 36$} & \multirow[t]{2}{*}{$0 \cdot 29$} \\
\hline & $81-254$ & 100-197 & $94-218$ & 105-191 & & & \\
\hline \multirow[t]{2}{*}{ Heart rate (beats per min) } & $90 \cdot 0(12 \cdot 7)$ & $91.4(8.4)$ & $88.0(9.9)$ & $89.5(12 \cdot 2)$ & \multirow[t]{2}{*}{$0 \cdot 80$} & \multirow[t]{2}{*}{0.52} & \multirow[t]{2}{*}{$0 \cdot 21$} \\
\hline & $70-118$ & $80-111$ & $70-100$ & $67-110$ & & & \\
\hline \multirow[t]{2}{*}{ White blood cell count $\left(\mathrm{mm}^{3}\right)$} & $13 \cdot 4(4 \cdot 5)$ & $11 \cdot 6(4 \cdot 7)$ & $10 \cdot 6(4 \cdot 5)$ & $11 \cdot 2(4 \cdot 2)$ & \multirow[t]{2}{*}{$1 \cdot 71$} & \multirow[t]{2}{*}{$0 \cdot 50$} & \multirow[t]{2}{*}{$0 \cdot 84$} \\
\hline & $6 \cdot 1-20 \cdot 1$ & $5 \cdot 6-9 \cdot 1$ & $5 \cdot 0-16 \cdot 6$ & $5 \cdot 3-15 \cdot 7$ & & & \\
\hline
\end{tabular}


Table 4 Relationships between sleep pattern and inflammatory response variables postCPB

\begin{tabular}{|c|c|c|c|c|c|c|c|c|c|c|c|c|}
\hline & \multicolumn{2}{|c|}{ Cortisol } & \multicolumn{2}{|c|}{$\begin{array}{l}\text { C-Reactive } \\
\text { Protein }\end{array}$} & \multicolumn{2}{|c|}{$\begin{array}{l}\text { Arterial Blood } \\
\text { Pressure }\end{array}$} & \multicolumn{2}{|c|}{ Blood Glucose } & \multicolumn{2}{|c|}{ Heart Rate } & \multicolumn{2}{|c|}{$\begin{array}{l}\text { White Blood } \\
\text { Cell }\end{array}$} \\
\hline & $r_{s}$ & $p$ & $r_{s}$ & $p$ & $r_{s}$ & $p$ & $r_{s}$ & $p$ & $r_{s}$ & $p$ & $r_{s}$ & $p$ \\
\hline Sleep onset latency & $0 \cdot 11$ & 0.629 & $0 \cdot 14$ & 0.542 & 0.03 & 0.906 & $-0 \cdot 39$ & $0 \cdot 114$ & 0.08 & 0.734 & -0.08 & 0.742 \\
\hline Wake after sleep onset & $0 \cdot 30$ & $0 \cdot 202$ & -0.28 & $0 \cdot 230$ & $-0 \cdot 02$ & $0 \cdot 906$ & 0.08 & 0.742 & 0.00 & 0.734 & 0.48 & 0.031 \\
\hline Sleep fragmentation index & 0.82 & $<0.001$ & 0.65 & 0.022 & $-0 \cdot 17$ & 0.491 & $-0 \cdot 23$ & $0 \cdot 349$ & $0 \cdot 14$ & $0 \cdot 547$ & 0.69 & 0.029 \\
\hline Total sleep time & -0.43 & 0.059 & 0.07 & 0.772 & -0.07 & 0.788 & 0.05 & $0 \cdot 863$ & $0 \cdot 04$ & $0 \cdot 872$ & $-0 \cdot 31$ & $0 \cdot 188$ \\
\hline Sleep efficiency index & $-0 \cdot 35$ & 0.128 & 0.58 & 0.015 & $-0 \cdot 11$ & 0.667 & 0.01 & 0.971 & -0.54 & 0.823 & -0.34 & $0 \cdot 144$ \\
\hline
\end{tabular}

Bold values indicate statistically significant.

of the directionality (e.g. bidirectional) of the relationship between sleep disturbance and inflammatory response is yet to be explored. Nevertheless, the data offer a platform for further empirical validation of the assumption that the degree of patients' sleep disturbances is proportional to the levels of stress and inflammation (i.e. systemic inflammatory response) postCPB, despite that the median salivary cortisol levels were within the normal range. Supporting this assumption and advancing the science in this field are the two notable findings emerging from this study. First, cardiac surgery patients are severely sleep-deprived during the immediate postoperative period, regardless of their pain and anxiety levels and the amount of medications consumed (Table 1). Most importantly, the effects of sleep-promoting (e.g. narcotic analgesics) and sleep-disrupting (e.g. betablockers) medications on patients' sleep patterns were not statistically significant, warranting further research in this patient population. Second, patients' sleep deprivation is associated with higher levels of inflammation and longer cross-clamp time.

\section{Degree of severity of sleep deprivation}

The severity of the patients' sleep deprivation is illustrated, in comparison with norms, by a marked increase in median WASO and SFI scores along with a marked decrease in the median TST and SEI scores across all postoperative nights (T1-T4). This degree of severity is further illustrated by nonsignificant changes in the effects of time on the median WASO, SFI, TST and SEI scores (Table 2). Compared with previous research, our study patients' sleep continuity (i.e. TST) and sleep efficiency (SEI) are worse. Pooled results from the four studies (Liao et al. 2011) that have used wrist actigraphy showed higher mean TST (5.0-5.9 hours) and SEI $(62 \cdot 6-63 \cdot 3 \%)$ compared with our data showing lower (poorer) mean TST and SEI scores of 2.6-4.8 (median 2.2-2.6) hours and 33.5-37.2\% (median 29-35), respectively. The differences in the findings are related to methodological issues and reflect differences in the measurement time periods, study settings and clinical variables, and two of the four studies were published more than a decade ago. Investigators of the three studies initiated the data collection during the second postoperative night, and the other investigator was not explicit about the time periods in which the sleep data were recorded during the 'first week' of hospitalisation. Notably, one of the four studies was implemented in Turkey (Yilmaz \& Iskesen 2007), which may have different patient care delivery processes than the USA. Thus, variations in care delivery processes in adult cardiac surgery programmes across countries, if any, and the extent to which these variations influence sleep outcomes require scrutiny. Another differentiating factor related to our data is the inclusion of 'off-pump' (CPB was not used) subjects in the two studies. It appears that patients who were not exposed to CPB machine have fewer sleep disruptions and higher sleep continuity than $\mathrm{CPB}$ patients (Liao et al. 2011).

In recent years, the comorbidities in the cardiac surgery population have become increasingly more complex (Bojar 2011). This trend may have also contributed to the degree of the severity of sleep disturbances/deprivation among the patients in the present study compared with those studies that were published a decade ago. We also found that the age of our study patients was negatively correlated with SOL $\left(r_{s}=-0.55, p=0.012\right)$. Changes in the sleep pattern occur as the person ages and the SOL prolongs (Vester et al. 2008, National Sleep Foundation 2010). Further research is needed to explore and understand the correlations between age and SOL postCPB due to the wide variations of the ranges in SOL scores found in the present study.

Despite the severity of our study patients' sleep deprivation, it is worth noting that their median SOL scores were within normal ranges ( $<15$ minutes) during postoperative nights 1 and 4, suggesting that the patients' propensity to fall asleep was quick. However, the SOL minimum and maximum scores ranged from $0-38$ minutes. This range in 
SOL scores is possibly related to small sample size, timing of data collection and variability in the timing and frequency of care provided to patients. At the onset of data collection (22:00 hours), the majority of the patients may have been asleep or simply immobile, thus providing low median SOL scores. On the contrary, a small number of patients may have required frequent vital signs and hemodynamic monitoring, turning to sides, blood sample draws and/or performing bedside diagnostic procedures at the start of sleep measurement (22:00 hours). Care delivery process is one of the main culprits causing sleep disruptions following cardiac surgery. A retrospective review of medical records reported by Tamburri et al. (2004) revealed a mean of 42.6 'care interactions' occurring between the hours of 19:00 and 07:00, with peak interactions during sleeping hours of 22:00 and 24:00 and early morning hours (07:00). Objective 'real-time' data, such as the use of cameras, in patient rooms are necessary to confirm the patient's activity at the onset of sleep measurement and the common clinical knowledge that patients' sleep disturbances are correlated with the nature of care delivery processes or care interactions. The majority of these studies, however, were implemented in noncardiac surgical units employing checklists and self-report questionnaires with inconclusive results (Casida \& Nowak 2011). Understanding the timing of the patient's sleep onset, whether it is influenced by movements initiated by the patient or induced by the healthcare provider, or extent to which the environments of care can influence the degree of sleep disturbances is critical knowledge gap that must be addressed to appropriately develop and test an intervention in postCPB patients.

\section{Sleep deprivation and exaggeration in inflammatory response}

Sleep deprivation is known to cause stress and inflammation in community-dwelling adults living with acute or chronic illnesses and traumatic conditions (Vester et al. 2008). In these populations, however, the relationships between sleep deprivation, SOL durations, C-RP and morning levels of cortisol are not clearly understood (Zhang et al. 2011). What is becoming clear is that sleep deprivation can exaggerate inflammation (Mullington et al. 2009, Patel et al. 2009). A systematic review of the sleep literature showed that a change in the person's sleep-wake cycle may be the first to trigger an acute state of inflammation (Ranjbaran et al. 2007). These findings are consistent with our data that demonstrated strong relationships between the variables of sleep deprivation (high SFI and WASO, and low SEI), stress (cortisol) and inflammation (C-RP and WBC). Whether these relationships are bidirectional, which means that sleep deprivation is linked with stress and inflammation and vice versa, postCPB is not known. Some studies have shown that sleep deprivation exaggerates inflammation and immune response, but the dysregulated immune system has protective and restorative effects during acute states (Ranjbaran et al. 2007). The increased C-RP (T2) and WBC (T1-T4) levels observed in the study patients may be suggestive of the acute systemic response during the immediate postCPB recovery period. Surgical incision, effects of sternal retractors and cross-clamp and the use of CPB have been reported as triggers for activating the inflammatory cascade, including the release of pro-inflammatory substances (cytokines) and leukocytosis manifested by an increased WBC level (Roth-Isigkeit et al. 2000, Raja \& Dreyfus 2005). This cascade is perhaps a contributing factor for the markedly increased SFI and WASO levels and markedly decreased TST and SEI levels of the patients in this study. Therefore, further research is needed to examine the extent of these relationships and investigate the role of pro-inflammatory markers (e.g. interleukins and tumour-necrotising factors) that may have mediating effects between sleep deprivation and inflammatory response postCPB.

Low SEI and sympathetic nervous system arousals (i.e. catecholamine release) manifested by increased HR level and high MAP are expected in sleep-deprived individuals (Hall et al. 2004) including canine models who have undergone CPB (Schuessler et al. 2012), a contrasting observation found in the present study warranting further explorations. Likewise, correlations between high WASO and longer cross-clamp time also require further explorations in future as there are no published data available for comparison. However, given the cross-clamp time of more than $60 \mathrm{~min}$ utes (Table 1), the ventricular function begins to deteriorate, leading to stimulation of the sympathetic nervous system (Bojar 2011). Stimulation of the sympathetic nervous system may be the contributing factor of frequent awakenings or longer WASO durations in the settings of normal HR and ABP across time periods (Tables 1 and 3). Nevertheless, the cardiac arrhythmia found in $30 \%$ of the subjects is a manifestation of sympathetic nervous system stimulation (Chelazzi et al. 2011); however, this explanation is inconclusive warranting further research.

\section{Limitations}

The main limitation of this study is the use of wrist actigraphy. The accuracy of wrist-actigraphically measured sleep relies on the wearer's hand movement. Some investigators have questioned the use of wrist actigraphy in critically ill 
patients based on the assumption that these patients have limited movements (Bourne et al. 2007), which may have explained the median SOL scores of 0 min throughout the study period. However, this assumption has not been validated objectively such as by the use of a camera or a direct observation involving large sample of critically ill patients including postCPB. In spite of the limitations, wrist actigraphy is used because it is practical and less invasive and does not interfere with patient care delivery in the intensive and progressive care units of hospitals (Casida \& Nowak 2011) as compared to using polysomnography, the gold standard for sleep measurement (Ancoli-Israel et al. 2003) designed for sleep laboratories. Thus, our data should be interpreted cautiously within the limits of wrist-actigrapically measured sleep patterns and preliminary (exploratory) studies. The methods we employed are subject to further refinement; therefore, our conclusions are not definitive and cannot be generalised.

\section{Future directions}

A set of investigative plans will be implemented in future to address the aforementioned study limitations and to further advance the science of sleep and inflammatory response in cardiac surgery. First, we will use a portable limited channel polysomnogram (i.e. home sleep testing monitor) to objectively measures sleep patterns, in which the data are not influenced by hand or body movement (Morales et al. 2012). Second, we will have larger sample size involving patients with or without CPB ('on or off pump'), secondtime cardiac surgeries ('redos' or 'reoperations') and other thoracic surgeries requiring $\mathrm{CPB}$ (e.g. aortic aneurysm repair) from different hospitals. Third, we will examine the effects of vasoactive agents (dose and frequency of administration), patient's menopausal status or menstrual cycle, and environmental factors (e.g. noise, light, temperature) on the sleep-wake patterns. Fourth, we will add pro-inflammatory marker (e.g. interleukins) variables and a subjective measure of sleep, which is an excellent source of elaborating sleep deprivation from patients' perspectives (Vester et al. 2008) to elicit comprehensive data on sleep and inflammation during the immediate postoperative period. Finally, we will employ an experimental design to determine the causal relationships between sleep disturbances, inflammatory response, postoperative complications and health outcomes.

\section{Conclusion}

Based on the findings of this study, patients who were exposed to $\mathrm{CPB}$ machine during cardiac surgery are severely sleep-deprived during the immediate postoperative period. The degree of the patients' sleep pattern disturbances or sleep deprivation was associated with biomarkers and surrogate markers of stress and inflammation. A larger sample is needed to validate these findings which are paramount for the development of a conceptual model to map out the underlying mechanisms of sleep pattern disturbances and inflammatory response postCPB. The model is crucial for developing and testing sleep-promoting interventions aimed to modulate the inflammatory response in cardiac surgery, leading to health promotion and reduction in risks and/or complications associated with CPB.

\section{Research and clinical implications}

In spite of the remarkable advancements in technology and refinement in surgical techniques over the past several decades (Bojar 2011), sleep pattern disturbances are prevalent in adult cardiac surgery (Liao et al. 2011). Nurses are well positioned to solve this chronic problem through the development and implementation of rigorous and methodologically sound studies based on the pathophysiology and mechanism of sleep pattern disturbances and associated outcomes postCPB. Based on the current state of the science related to sleep in cardiac surgery (Casida \& Nowak 2011, Liao et al. 2011), it is premature for nurse-scientists to delve into designing and testing interventions. At this stage, research should be focused on understanding the mechanisms of the clinical phenomena with research designs that address the directionality and causality of the nature of the relationship between the degree of sleep disturbances and inflammatory response in adult cardiac surgery. In the absence of this knowledge, the validity of the results and the likelihood of producing negative results of interventions cannot be avoided (Casida \& Nowak 2011).

Sleep promotion is a nurse-driven intervention consistent with the basic understanding about the interactions of health and environment, dating back to the era of Florence Nightingale (Fawcett \& Desanto-Madeya 2012). Cardiac surgery nurses have the power to transform the care processes and the environments of care that presumably affects the sleep patterns of patients during the immediate recovery period. However, nurses must be cognizant about manipulating the care delivery process without substantive evidence to support the change in practice. Otherwise, nurses will not be successful in making a case to create policy and influence the multidisciplinary healthcare team to change the night-time routine care delivered to patients in the cardiac surgical units. Nurses must lead the efforts in advancing the science of sleep and sleep outcomes among 
hospitalised patients. Additionally, nurses must show the public that these efforts are directly related to their roles in health promotion and risk reduction. Interventions targeting variables that disrupt the sleep-wake cycle can help modulate the inflammatory response which may reduce inflammation-associated complications in cardiac surgery.

\section{Acknowledgements}

The authors thank the following for their support in the implementation and completion of this study: Anna Marrocco, MSN, APRN-BC, Geri Babicz, BSN, RN, Monica Elia, BSN Student, Mahwish Hafeez, BS, Lab Technician, Danny St. Louis, MSN, APRN-BC, the P5, I5, and H5 nursing staff, and Cardiothoracic Surgeons at Henry Ford Hospital in Detroit, Michigan, USA.

\section{Disclosure}

The authors have confirmed that all authors meet the ICMJE criteria for authorship credit (www.icmje.org/ethical_1author.html), as follows: (1) substantial contributions to conception and design of, or acquisition of data or analysis and interpretation of data, (2) drafting the article or revising it critically for important intellectual content, and (3) final approval of the version to be published.

\section{Funding}

This study was partially funded by the Robert Wood Johnson Foundation Nurse Faculty Scholars Program Grant \# 66525 and supported by the Wayne State University College of Nursing Biophysical Laboratory.

\section{References}

Ancoli-Israel S, Cole R, Alessi C, Chambers M, Moorcoft W \& Pollack PP (2003) The role of actigraphy in the study of sleep and circadian rhythms. Sleep 26, 342-392.

Aubert-Tulkens G, Culee C, Harmant-Van Rijckevorsel K \& Rodenstein DO (1987) Ambulatory evaluation of sleep disturbance and therapeutic effects in sleep apnea syndrome by wrist activity monitoring. American Review of Respiratory Disease 136, 851-856.

Bojar RM (2011) Manual of Perioperative Care in Adult Cardiac Surgery, 5th edn. Wiley-Blackwell, West Sussex, UK.

Bourne RS, Minelli C, Mills GH \& Kandler R (2007) Sleep measurement in critical care patients: research and clinical implications. Critical Care 11, 226.

Cappabianca G, Paparella D, Visicchio G, Capone G, Lionetti G, Numis F, Ferrara P, D'Agostino C \& de Luca Tupputi Schinosa L, (2006) Perioperative C-reactive protein predicts mid-term outcome after cardiac surgery. Annals of Thoracic Surgery 82, 2170-2178.

Casida JM \& Lemanski S (2010) An evidence-based review on guided imagery utilization in adult cardiac surgery. Clinical Scholars Review 3, 22-26.

Casida JM \& Nowak L (2011) Integrative therapies to promote sleep in the intensive care unit. In Integrated Therapies in Lung Health or Sleep
(Chlan L \& Hertz M eds). Springer, New York, NY, pp. 177-187.

Casida JM, Davis JE, Brewer RJ, Smith C \& Yarandi H (2011) Sleep and daytime sleepiness of patients with left ventricular assist devices: a longitudinal pilot study. Progress in Transplantation 21, 131-136.

Chelazzi C, Villa G \& DeGaudio AR (2011) Postoperative atrial fibrillation. ISRN Cardiology 2011, 203179.

Christ-Crain M, Jutla S, Widmer I, Couppis O, König C, Pargger H, Puder J, Edwards R, Müller B \& Grossman AB (2007) Measurement of serum free cortisol slows discordant responsivity to stress and dynamic evaluation. The Journal of Clinical Endocrinology \& Metabolism 92, 1729-1735.

Estrada-Y-Martin RM \& Orlander PR (2011) Salivary cortisol can replace free serum cortisol measurements in patients with septic shock. Chest 140, 1216-1222.

Fawcett J \& DeSanto-Medeya S (2012) Contemporary nursing knowledge: analysis and evaluation of nursing models and theories, 3rd edn. F.A Davis, Philadelphia, PA.

Hall M, Vasko R, Buysee D, Ombao H, Chen Q, Cashmere JD, Kupfer D \& Thayer JF (2004) Acute stress affects heart rate variability during sleep. Psychosomatic Medicine 66, 56-62.

Hoda MR, El-Achkar H, Schmitz E, Scheffold $\mathrm{T}$, Vetter $\mathrm{HO}$ \& DeSimone $\mathrm{R}$
(2006) Systemic stress hormone response in patients undergoing open heart surgery with or without cardiopulmonary bypass. Annals of Thoracic Surgery 82, 2179-2186.

Liao WC, Huang CY, Huang TY \& Hwang SL (2011) A systematic review of sleep patterns and factors that disturb sleep after heart surgery. Journal of Nursing Research 19, 275-288.

Morales CR, Hurley S, Wick LC, Staley B, Pack FM, Gooneratne NS, Maislin G, Pack A \& Gurubhagavatula I (2012) In-home, self-assembled sleep studies are useful in diagnosing sleep apnea in the elderly. Sleep 11, 1491-1501.

Mullington JM, Haack M, Toth M, Serrador J \& Meir-Ewert HK (2009) Cardiovascular, inflammatory and metabolic consequences of sleep deprivation. Progress in Cardiovascular Diseases 51, 294-302.

Mullington JM, Simpson NS, Meier-Ewer HK \& Haack M (2010) Sleep loss and inflammation. Best Practices Research: Clinical Endocrinology \& Metabolism 24, 775-784.

Munro BH (2001) Statistical Methods for Health Care Research, 4th edn. Lippincott, Philadelphia, PA.

National Sleep Foundation (2010) Sleep and Ethnicity. National Sleep Foundation, Arlington, VA. Available at: http://www.sleepfoundation.org/article/ sleep-america-polls/2010-sleep-and-ethnicity (accessed 8 April 2013). 
Parish JM (2009) Sleep-related problems in common medical conditions. Chest 135, 563-572.

Patel SR, Zhu X, Storfer-Isser A, Mehra R, Jenny NS, Tracy S \& Redline S (2009) Sleep duration and biomarkers of inflammation. Sleep 32, 200 204.

Raja SG \& Dreyfus GD (2005) Modulation of systemic inflammatory response after cardiac surgery. Asian Cardiovascular \& Thoracic Annals 13, 382-395.

Ranjbaran Z, Keefer L, Stepanski E, Farhadi A \& Keshavarzian A (2007) The relevance of sleep abnormalities to chronic inflammatory conditions. Inflammation Research 56, 51-57.

Rao NL, Shetty S, Upadhyaya K, Prasad RM, Lobo EC, Kedilaya HP \& Prasad G (2010) Salivary C-reactive protein in Hashimoto's Thyroiditis and Subacute Thyroiditis. Interna- tional Journal of Inflammation 2010, 514659.

Respironics (2008) Actiware and Actiware CT Software Manual. Respironics, Bend, OR.

Roth-Isigkeit A, Dibbelt L, Schmucker P \& Seyfarth M (2000) The immune-endocrine interaction varies with the duration of the inflammatory process in cardiac surgery patients. Journal of Neuroendocrinology 12, 546-552.

Salimetrics (2009) Saliva Collection and Handling Advise. Salimetrics, State College, PA.

Salimetrics (2010) Salivary C-Reactive Protein ELISA Kit. Salimetrics, State College, PA.

Schuessler RB, Ishii Y, Khagi Y, Diabagate K, Boineasu JP \& Damiano RJ (2012) The effects of inflammation on heart rate and rhythm in a canine model of cardiac surgery. Heart Rhythm 9, 432-439.
Tamburri LM, DiBrienza R, Zozula R \& Redeker NS (2004) Nocturnal care interactions with patients in critical care units. American Journal of Critical Care 13, 102-112.

Vester JC, Pandi-Perumal SR \& Streiner DL (2008) Sleep and Quality of Life in Clinical Medicine. Humana Press, Totowa, NJ.

Yilmaz H \& Iskesen I (2007) Follow-up with objective and subjective tests of the sleep characteristics of patients after cardiac surgery. Circulation Journal 71, 1506-1510.

Zhang J, Ma RCW, Kong APS, So WY, Li AM, Lam SP, Li SX, Yu MW, Ho CS, Chan MH, Zhang B \& Wing YK (2011) Relationship of sleep quantity and quality with 24-hour urinary catecholamines and salivary awakening cortisol in healthy middle-aged adults. Sleep 34, 225-233.

The Journal of Clinical Nursing (JCN) is an international, peer reviewed journal that aims to promote a high standard of clinically related scholarship which supports the practice and discipline of nursing.

For further information and full author guidelines, please visit JCN on the Wiley Online Library website: http:// wileyonlinelibrary.com/journal/jocn

\section{Reasons to submit your paper to JCN:}

High-impact forum: one of the world's most cited nursing journals, with an impact factor of 1.316 - ranked 21/101 (Nursing (Social Science)) and 25/103 Nursing (Science) in the 2012 Journal Citation Reports ${ }^{\circledR}$ (Thomson Reuters, 2012).

One of the most read nursing journals in the world: over 1.9 million full text accesses in 2011 and accessible in over 8000 libraries worldwide (including over 3500 in developing countries with free or low cost access).

Early View: fully citable online publication ahead of inclusion in an issue.

Fast and easy online submission: online submission at http://mc.manuscriptcentral.com/jcnur.

Positive publishing experience: rapid double-blind peer review with constructive feedback.

Online Open: the option to make your article freely and openly accessible to non-subscribers upon publication in Wiley Online Library, as well as the option to deposit the article in your preferred archive. 